\title{
La formación de usuarios con metodos participativos para estudiantes universitários
}

\section{Saray Córdoba González}

\section{Resumen}

En este artículo se plantea cómo el método que se aplique en la formación de usuarios de la información, está estrechamente relacionado con el éxito que se obtenga. Con este $y$ otros argumentos se justifica la aplicación del método participativo, destacando los resultados alcanzados en su aplicación en la experiencia obtenida en la Universidad de Costa Rica. Para ello se presenta inicialmente una revisión conceptual de los distintos términos que se han utilizado, insistiendo en la necesidad de alcanzar como meta la formación de una cultura de información en los países de América Latina.

\section{Palabras-clave}

Formación de usuarios; Cultura de información; Método participativo en la formación de usuarios; Usuario de la información.

\section{INTRODUCCION}

La formación de usuarios es una tarea que los profesionales de la información deben enfrentar en los diferentes niveles del sistema educativo formal, cuyas consecuencias se pueden observar posteriormente a través de toda la vida de la persona, como usuario de la información. Sin embargo, ésta es una tarea que muchas veces es evadida por cuanto es preferible dedicarse a las actividades que deparen resultados más inmediatos o aquellas que necesiten una urgente atención.

Mientras tanto, la ausencia de hábitos de lectura en la población latinoamericana, la falta de buenas y suficientes bibliotecas escolares, públicas e infantiles, la existencia de un profesional de la información caracterizado por ser pasivo y la escasa conciencia sobre la importancia de la información en diferentes niveles de la población adulta, provocan que el uso de la información sea muy escaso y casi ignorado.

Pero si se reconoce que la formación de usuarios constituye un aporte muy valioso al desarrollo de una nación o comunidad, podremos observar que sus efectos a largo plazo, que son el resultado de nuestra acción profesional, serán contundentes para lograr que las personas lleguen a ser más productivas, reflexivas y capaces de explotar más eficientemente su inteligencia.

La práctica demuestra sin embargo, que ha sido común que sea en las bibliotecas universitarias donde exista la preocupación por formar al usuario, pues allí se debe enfrentar corrientemente la incómoda situación de verse obligados a explicarles repetitivamente el uso y la explotación de los recursos que ella ofrece. Dado que la mayoría de las unidades de información en la región latinoamericana son limitadas en recursos, es allí donde el estudiante encuentra la tecnología de información que nunca antes había conocido, y por ello es en ese nivel que se vuelve indispensable trabajar con un usuario conocedor del manejo de todos esos instrumentos de búsqueda de la información.

Por ello, la tendencia predominante es la de enfrentar la emergencia, pues se necesita enseñarle urgentemente a aquel estudiante a utilizar el disco compacto que nunca ha visto en su vida, o a manejar un índice que aun se publica en papel, cuya estructura se presenta extraña y complicada a unos ojos que nunca han conocido algo igual. Así, los esfuerzos realizados en ese sentido se han caracterizado más por la instrucción que por la educación de los usuarios, sin lograr que éstos lleguen a estar conscientes de las ventajas que les ofrece el uso y la explotación de los recursos informativos.

En este trabajo se plantea cómo el método que se aplique en la formación de usuarios de la información, está estrechamente relacionado con el éxito que se obtenga. Por ello se ofrecen los resultados alcanzados en la aplicación del método participativo en este campo, según la experiencia relatada en una reciente publicación (Córdoba, 1996). Para ello desarrollo inicialmente una revisión conceptual de los distintos términos que se han utilizado, insistiendo en la necesidad de alcanzar como meta la formación de una cultura de información en los países de América Latina. 


\section{REVISION CONCEPTUAL}

Existen al menos tres matices diferentes que en la práctica ha presentado el concepto de la formación de usuarios.

El primero y más común, es el que tiene un objetivo utilitario - como es el caso del contenido del término instrucción el cual se aplica cuando se desea que el usuario aprenda el manejo de las fuentes de información, tradicionalmente denominadas "abstracts" o índices, publicadas en formato digital o impresas; las bases de datos o el catálogo en línea (OPAC).

En este caso, cabe señalar aquí la definición de Tessier (cfr. Dupont, 1992) quien destaca que ésta es...

el conjunto de actividades de aprendizaje que permiten conocer y utilizar las fuentes de información y los recursos documentales óptimos, a fin de responder a las necesidades de información para fines de estudio, de investigación y de actualización permanente.

Se observa que su sentido se limita a que el usuario conozca los instrumentos que tiene la unidad de información, con el fin de que sean utilizados correctamente. Así, el profesional de la información economizará tiempo y esfuerzo al evitar la repetición innecesaria de la misma instrucción a diversos usuarios, durante múltiples veces. Las sesiones o jornadas a usuarios se realizan generalmente, utilizando módulos de autoinstrucción, medios audiovisuales o multimedios y en los casos más comunes, folletos o plegables complementados con charlas.

Esta misma concepción ha evolucionado hasta llegar más recientemente a lo que algunos autores han denominado como "alfabetización en información" , aunque se diferencia de su antecesora porque ésta se inicia desde la escuela, o como lo expone Fjällbrant (1996), provee al estudiante de "conceptos y herramientas que serán útiles, no solo en conexión con sus estudios inmediatos, sino a través de toda su vida laboral". No obstante, esta idea mantiene ese sentido utilitario que mencioné antes, pues no trasciende el objetivo de desarrollar una habilidad en el sujeto para que use las fuentes y los productos de información.
El segundo concepto es aquel que asimila la formación de usuarios con la divulgación o mercadeo de los servicios y productos que ofrece la unidad de información y que en el pasado se llamó también atracción de usuarios. Así, se distribuyen materiales impresos que contienen instrucciones e información sobre el contenido de la unidad de información - por ejemplo - los nuevos servicios que ofrecen o los nuevos instrumentos que se adquirieron. El uso de plegables, boletines o trípticos es común en estos casos, así como los vídeos que se transmiten en forma continua, y los "homepages" que existen en la red, los cuales se distribuyen masivamente como medio para mercadear la oferta.

El tercer concepto es aquel que tiene un objetivo más integral, permanente y profundo, al que Wilson (1980) le ha llamado educación de usuarios y pretende que:

los usuarios potenciales de la información, - los encargados de elaborar políticas nacionales en esta esfera, adquieran conciencia del valor de la información para actividades especializadas, adopten actitudes positivas con respecto a la necesidad de buscar información y estén motivados para utilizar o desarrollar recursos informativos.

Tomar conciencia no es una tarea fácil, pero paulatinamente se deberá romper el círculo vicioso en el cual han caído los países pobres en información. Si no somos capaces de usar la información, tampoco podremos producirla y mucho menos, promover su uso mediante políticas nacionales que se diseñen con ese cometido.

Tratando de actualizar este concepto, se desprende que el objetivo a largo plazo de las unidades de información - sobre todo de la educación formal - ha de ser formar una cultura de información, definida por Menou (1996, p. 298) como "la habilidad de los individuos o grupos de hacer el mejor uso posible de la información". Así, se debe educar al usuario en las etapas tempranas de su vida para lograr un adulto conciente de la importancia del uso de la información y consecuentemente, que esté interesado en promoverla y generarla.
Se trata de un proceso que se inicia desde que la persona nace, cuando se promueve la lectura por medio de los cuentos y fábulas en la primera infancia. Continúa en la escuela, donde no solo se promueve la lectura sino además se forma al niño hacia el uso de los instrumentos que permitan explotar la información, tales como el catálogo, los multimedios, las obras de referencia y el libro en todos sus diferentes soportes.

Ya en la universidad, los profesionales de la información se dedicarán a demostrar los beneficios de la información, a partir del apoyo que se ofrezca al estudiante que sabe explotar las fuentes y recursos que contiene la unidad de información y utilizar adecuadamente la tecnología que tenga a su alcance.

El resultado de este proceso será un adulto educado, capaz de tomar decisiones fundamentadas, capaz de explotar su propio potencial y consecuentemente, más productivo y con capacidad para aportar al desarrollo nacional. Asimismo, tendrá mayor potencial mental al concentrarse en saber "cómo encontrar el conocimiento dondequiera que esté, en lugar de tratar de recordar todo cuanto haya aprendido" Éste es precisamente el concepto de "aprender a aprender" que la Unesco ha promovido a partir de 19 .

Así, el concepto de cultura de información va más allá del simple entrenamiento para desarrollar habilidades; por el contrario, ha de darse como resultado de un proceso sistemático y prolongado; una combinación de experiencias individuales y colectivas, hasta llegar a formar parte de la herencia cultural (Menou, 1996).

De aquí que de estas tres concepciones, y tomando en cuenta las características que presenta la región latinoamericana, es conveniente e ideal desarrollar estrategias de educación de usuarios, que sean permanentes y que se den en diferentes niveles, de manera que se logre un proceso continuo y sistemático que sea paralelo al proceso de formación del ser humano, aplicando los métodos más adecuados en cada nivel. 


\section{LAS ESTRATEGIAS METODOLOGICAS}

En términos ideales la formación de una cultura de información debe ser la meta de los especialistas de la información. Sin embargo, las condiciones socioeconómicas de la región latinoamericana, y más ampliamente de los países pobres del globo, obligan a abrir los ojos ante la falta de hábitos de lectura y la escasa conciencia por parte de los docentes, los políticos o los planificadores, sobre la importancia que tiene la información en su trabajo o en su vida cotidiana.

Así, no se puede aspirar a enfrentarnos comúnmente a ese usuario educado, sino que la realidad en la que estamos inmersos exige atender a un usuario generalmente sin formación, que pocas veces se ha enfrentado a los instrumentos de búsqueda que tiene una biblioteca, por más sencillos que éstos sean. La deficiencia de las bibliotecas escolares, las escasas acciones que se ponen en práctica para promocionar la lectura o la información en edades tempranas, la poca importancia que se le ha asignado al usuario en la formación del bibliotecario y la prevalencia de métodos educativos tradicionales, que ponen énfasis en la memorización, la pasividad y la actitud bancaria - muy utilizados aún en las instituciones educativas básicas de América Latina - contribuyen efectivamente al poco uso de la información.

De esta manera se observa que la situación no es sencilla. Sus múltiples aristas nos llevan a pensar que los sujetos de las acciones que se emprenden, también han de ser variados: los docentes y los estudiantes en diferentes niveles, los políticos, los que toman las decisiones, los dirigentes de organizaciones y muchos más. Sin embargo, ante la incapacidad de abarcar simultáneamente toda esa gama de sujetos en los esfuerzos que se realicen, es necesario priorizar y para ello definir políticas y estrategias adecuadas para cada nivel.
Esta complejidad a su vez permite observar claramente un cometido insoslayable en las acciones que se emprendan. Éste es la necesidad de enfatizar en la toma de conciencia de los usuarios acerca de la importancia de la información en todas las actividades que emprendan, y no solo en su entrenamiento para el uso de los instrumentos de búsqueda o productos. Llegar a un usuario convencido de que la información es un recurso cuya tenencia genera poder, cuya explotación acarrea múltiples ventajas, por lo que hay muchas razones en las que reside su valor y se justifica su utilización, ha de ser la meta que oriente todas las acciones y proyectos que se desarrollen en este campo en cualquie unidad de información.

Sin embargo ésta no es una meta sencilla, pues para crear conciencia sobre las ventajas que tiene el uso y la explotación de los recursos informativos, se requiere diseñar una estrategia que sea pertinente para el tipo de usuario, sistemática y permanente. Se deben utilizar métodos activos o participativos y claramente delineados en cuanto a los pasos que los conforman: El punto de partida y de llegada, las técnicas y los objetivos deberán formar un todo indivisible y compacto que tienda a lograr el cometido propuesto.

En el caso de los estudiantes universitarios, ubicados como adultos jóvenes o en su última etapa de la adolescencia, es importante que la formación de usuarios se ofrezca en el momento adecuado, cuando se presente la necesidad inmediata según las exigencias de su plan de estudios. También es importante tomar en cuenta que no es una persona fácilmente manipulable ya que su capacidad de crítica y razonamiento impide que se le puedan imponer ideas o costumbres. El conocimiento que se le ofrezca debe ser útil para él o ella en ese momento y se debe partir de una base llena de experiencias previas, por lo que tiene que existir una motivación especial para aceptar la jornada de formación de usuarios que se le proponga.
El bagaje de conocimientos y experiencias que tiene el usuario ha de ser determinante para escoger el método más adecuado, de manera que éste sea el sujeto de su propio proceso de aprendizaje y no un observador pasivo. Además, su nivel de participación debe permitirle decidir sobre lo que debe hacer para actuar conscientemente de manera que posterior al proceso de formación, sus actos estén mediados por su razón e inteligencia. Así, se debe pretender la toma de conciencia sobre la existencia de una necesidad de información para transformar su comportamiento hacia ella.

Es por estas razones que los métodos coercitivos generalmente no funcionan. Por ejemplo, solicitar que el estudiante atienda a un curso como condición para entregarle su carné de usuario o que una charla sobre los servicios de la biblioteca es indispensable para autorizarle el préstamo a domicilio, son formas equivocadas que producen el rechazo de los estudiantes. Al respecto Weaver (1997) expone su preocupación de la siguiente manera: "Ofrecer la instrucción al usuario desconectada a un curso particular, puede ser como tener una fiesta a la cual nadie acude". De igual manera, algunas evaluaciones que se han realizado, citadas por Tiefel (1995, p. 325) plantean críticas a los cursos formales o a las sesiones generales porque "trivializan la recolección de la información".

Se podría agregar que este rechazo sucede no solo en relación con la coerción que se aplique, sino también con las otras condiciones que ya fueron enumeradas anteriormente. Todas ellas pertenecen a la cultura, que es el bagaje que la persona adulta ha acumulado durante su vida, a la que Menou (1996) le asigna el calificativo de "soberana". 


\section{EL METODO PARTICIPATIVO}

La toma de conciencia sobre la importancia de la información debe pasar por un ciclo de conocimiento holístico que no haga diferencia entre la fase de adquisición del conocimiento existente y la fase de la creación de nuevo conocimiento. Romper esa dicotomía es indispensable para alcanzar un proceso educativo transformador y creativo. Para ello es necesario lograr una postura crítica de parte de los sujetos cognoscentes, ante la realidad, postura que según Freire (1986, p. 83)...

es negada cada vez que, rompiéndose la relación dialógica, se instaura un proceso de pura transferencia de conocimiento, en el cual conocer deja de ser un acto creador y recreador para ser un acto "digestivo".

Lo anterior quiere decir que si se utilizan métodos tradicionales - propios de la educación bancaria - para formar a un usuario que debe tomar conciencia de que la información es fundamental para su quehacer, no es posible lograrlo por más técnicas novedosas e información impresa que se utilice. El método debe ir de acuerdo con las metas que se hayan trazado, con las características de los sujetos cognoscentes y con el contexto en el cual se desenvuelven esos sujetos.

Ahora bien, de esta toma de conciencia se pretende un cambio de actitud hacia el uso de la información la cual será la meta del proceso educativo al cual nos enfrentemos. Si se enfrenta a un usuario poco habituado a hacer uso de los recursos de información, con escaso hábito de lectura y consecuentemente, con cierta actitud negativa hacia todo lo que se refiera a la información, entonces es conveniente aplicar un método que se adecue a estas características, las cuales describí con más detalle en el apartado anterior.

El método participativo está fundamentado en el método dialéctico que se ha aplicado a la educación con la finalidad de transformar la realidad de los sujetos. Su valor fundamental se basa en la creatividad productiva que tienen los sujetos y por ello la enseñanza se centra en las necesidades concretas del individuo o grupo.
De aquí que es indispensable partir de un triple diagnóstico que permita conocer el entorno institucional, las características del usuario y sus experiencias previas en el uso de la información. Así, se parte de los problemas del grupo, de manera que no es posible llegar con ideas preestablecidas ni llevar material prefabricado, sino que las técnicas deben tener tal flexibilidad, que permitan variaciones durante el proceso, previendo un mínimo de condiciones a partir del diagnóstico citado.

Las relaciones entre los sujetos - el facilitador y el cognoscente - deben ser de intercambio, plenas relaciones horizontales en donde la realidad por transformar, se encuentre en el medio de esa relación (López, 1989, p. 34). De aquí que se parte del supuesto de que el usuario tiene tanto que enseñar como los facilitadores de aprender de él; precisamente ésa es la relación dialógica a la que se refiere Freire, y que fue citada anteriormente.

No se puede obviar que la aplicación de los tres momentos del método participativo - partir de la práctica, teorizar y volver a la práctica - son las fases fundamentales que enmarcan las técnicas, los objetivos, las temáticas y los procedimientos. En este sentido, tanto las experiencias desarrolladas con los estudiantes de primer ingreso (Córdoba, 1996), como con los estudiantes de la Carrera de Trabajo Social (Rodríguez y Arguedas, 1995), ambos de la Sede de Occidente, de la Universidad de Costa Rica, demostraron que es viable su aplicación, siempre que se dé formando un todo indisoluble y abarcando las características descritas anteriormente. En ambos casos se relatan y evalúan las experiencias que se tuvieron al aplicar el método participativo para la formación de usuarios, con estudiantes de una universidad costarricense. En ellos se describen detalladamente las técnicas y los procedimientos que se aplicaron y los resultados obtenidos.

\section{REFLEXIONES GENERALES}

Posterior a las experiencias obtenidas en la aplicación y revisión del método participativo, han ido surgiendo algunas reflexiones que me parece importante compartir. Éstas están basadas en la observación de los cambios que se han efectuado en los actores sociales de la Sede de Occidente (UCR), en diferentes momentos y espacios, después de varios años de ocurrida esta experiencia. Éstas son:

1. La necesidad de realizar cambios curriculares en la formación del profesional de la información. - Cada vez parece más importante reforzar el área de los usuarios en los currícula, de manera que el graduado no solo egrese con mayor conciencia sobre la importancia que éstos tienen, sino que obtenga la formación básica en cuanto a los métodos educativos que puede aplicar. La experiencia costarricense demuestra que esa área es muy débil en los currícula pues no existen cursos de educación de usuarios específicamente, sino que los respectivos contenidos son compartidos con otros temas relacionados.

2. La necesidad de ofrecer capacitación a los profesionales de la información en servicio. - La situación descrita anteriormente justifica la necesidad de capacitar a los profesionales en servicio sobre los conceptos, métodos y técnicas que se aplican, y sobre la planificación de proyectos de formación de usuarios. Los vacíos que existen en este campo deben atacarse de manera eficaz, pues solo así será posible que la educación de los usuarios sea una realidad, dentro de la concepción que expuse en el primer apartado.

3. Se han observado algunas variaciones en las prácticas profesionales. - Es posible avizorar un nuevo papel para el profesional de la información, no solo porque así ha ido variando en la percepción de los usuarios, sino porque los mismos profesionales se observan a sí mismos diferentes. Estos agentes sociales que se han involucrado en las experiencias que se desarrollaron en la Sede de Occidente, y que fueron citadas anteriormente, han reforzado su papel participativo en los diferentes niveles en que actúan, de manera que sus 
exigencias en ese plano son mayores. También ha variado su trato con el usuario, producto de una percepción diferente hacia él: de un usuario-objeto, se varía hacia un sujeto social, lo cual tiene consecuencias muy positivas para la práctica del profesional de la información.

También me ha sido posible observar en mi práctica docente, una reconceptualización por parte de los usuarios, acerca de la biblioteca. Los estudiantes han expresado que su percepción hacia ella cambió después de asistir a la jornada que se les ofreció y ha crecido el índice de utilización de los servicios y productos de la biblioteca.

4. Se ha generado un debate latente respecto al manejo de la reglamentación en la Biblioteca. - También en este campo algunos de los profesionales de la información que participaron en estas experiencias se han vuelto más flexibles en la aplicación de las normas que rigen los servicios. La incongruencia entre los postulados teóricos que plantea la metodología participativa y las viejas normas que rigen la organización de las relaciones entre los actores sociales involucrados, ha generado una discusión entre los profesionales, cuyo desenlace aún es difícil de vislumbrar. Lo que sí es observable es que existe un cambio de actitud en este sentido, de manera que

\section{Higher user education active methods}

\begin{abstract}
This paper justify how the user education method is giving rise to success in the information area. A conceptual description is first presented discussing the different approaches used for this concept within diverse spheres. The attention should be paid to the systematic and permanent education effort in order to create an information culture for Latin American countries. The active method proved at the Universidad de Costa Rica seems to be the best choice with higher education students. Conclusions are drawn regarding the UCR experience after several years of trying this method.
\end{abstract}

\section{Keywords}

User education; Information and culture; Active method in user education; Information user. el usuario recupera su papel preponderante en la dinámica de la biblioteca.

5. Se ha sentido la necesidad de promover proyectos prácticos que permitan evaluar las experiencias de formación de usuarios. - En este sentido es necesario desarrollar mayores aplicaciones con otros grupos de usuarios. Por ejemplo, hemos empezado a desarrollar un plan de capacitación dirigido a bibliotecarios escolares, con el fin de aplicar el método participativo a estudiantes de secundaria. Posteriormente evaluaremos la experiencia y valoraremos la factibilidad de aplicar el método a ese nivel.

6. Es necesario teorizar y retroalimentar los postulados teóricos-metodológicos de la formación de usuarios. - Cada vez que se apliquen experiencias novedosas, se debe sistematizar la experiencia para que otros profesionales analicen la posibilidad de aplicarla en su campo. Para ello, es muy importante publicar los resultados y dar a conocer por otras vías los procedimientos que tuvieron éxito. De aquí podremos desarrollar una teoría adecuada a las necesidades de la gran patria que es América Latina.

Finalmente es oportuno repasar las palabras de Paulo Freire que vienen a mi memoria a propósito de los métodos educativos:

Tenemos que respetar los niveles de comprensión que los educandos - no importa quiénes sean - están teniendo de su propia realidad. Imponerles nuestra comprensión en nombre de su liberación es aceptar soluciones autoritarias como caminos de libertad.

\section{REFERÊNCIAS BIBLIOGRÁFICAS}

1. CÓRDOBA, Saray. Formación de usuarios para estudiantes universitarios: una experiencia con metodología participativa. México, D.F.: UNAM, CUIB, 1996. 30 p.

2. DUPONT, Lisette. "La place de la formation documentaire dans la formation universitaire". Documentation et bibliothèques, 38(2):-35-40, Jan-Mar, 1992.

3. FJÄLLBRANT, Nancy. "EDUCATE: a networked user education project in Europe". IFLA Journal, 22(1):31-34, 1996.

4. FREIRE, Paulo. La importancia de leer y el proceso de liberación. 4a. ed. México Siglo XXI, 1986.

5. HERNÁNDEZ, Patricia y otras. Seminario latinoamericano sobre formación de usuarios de la información y los estudios de usuarios. México D.F.: UNAM, Centro Universitario de Investigaciones Bibliotecológicas, 1997.

6. LÓPEZ DE CEBALLOS, Paloma. Un método para investigación-acción participativa. Madrid: Ed. Popular, 1989.

7. RODRÍGUEZ, Alba y Arguedas, Ana J. Estrategia de formación de usuarios utilizando metodología participativa. Caso: estudiantes de Trabajo Social en la Sede de Occidente. (Tesis Lic. Bibliotecología). Ciudad Universitaria Rodrigo Facio: Universidad de Costa Rica, Escuela de Bibliotecología y Ciencias de la Información, 1995.

8. SAULE, Mara R. "User instruction issues for databases in the Humanities". Library Trends, 40(4):596-613, 1992.

9. TIEFEL, Virginia M. "Library use education: examining its past, projecting its future". Library trends, 44(2):318-338, 1995.

10. WEAVER, Patricia. "Marketing library instruction to adults". College and Research Libraries News, 58(7):493-494, 1997.

11. WILSON, T.D. Directrices para el planeamiento y ejecución de un plan nacional de formación y enseñanza para el uso de la información. París: UNESCO, 1980.

Artigo aceito para publicação em 01-4-98.

Saray Córdoba González

Magister Scientiae em educação de adultos. Licenciada em bibliotecologia e ciências da informação. Cadedrática pela Universidade de Costa Rica. Atual diretora do Centro de Informação e Referência sobre a América Central e Caribe (Circa).

E-mail: scordoba@cariari.ucr.ac.cr 